\title{
Phantom-based quantification of the spectral accuracy in dual-layer spectral CT for pediatric imaging at $100 \mathrm{kVp}$
}

\author{
Sebastian Meyer ${ }^{1,{ }^{*}}$, Leening P. Liu ${ }^{1}$, Harold I. Litt ${ }^{1}$, Sandra S. Halliburton ${ }^{2}$, Nadav Shapira $^{1}$, Peter B. Noël ${ }^{1,3}$ \\ ${ }^{1}$ Department of Radiology, Perelman School of Medicine, University of Pennsylvania, Philadelphia, PA 19103, USA \\ ${ }^{2}$ Philips Healthcare, Orange Village, OH 44122, USA \\ ${ }^{3}$ Department of Diagnostic and Interventional Radiology, School of Medicine \& Klinikum rechts der Isar, Technical \\ University of Munich, 81675 München, Germany \\ *Now at: Department of Medical Physics, Memorial Sloan Kettering Cancer Center, New York, NY 10065, USA
}

\begin{abstract}
Objectives To determine the spectral accuracy in detector-based dual-energy CT (DECT) at $100 \mathrm{kVp}$ and wide $(8 \mathrm{~cm})$ collimation width for dose levels and object sizes relevant to pediatric imaging.

Methods A spectral CT phantom containing tissue-equivalent materials and iodine inserts of varying concentrations was scanned on the latest generation detector-based DECT system. Two 3D-printed extension rings were used to mimic varying pediatric patient sizes. Scans were performed at 100 and $120 \mathrm{kVp}, 4$ and $8 \mathrm{~cm}$ collimation widths, and progressively reduced radiation dose levels, down to $10 \%$ of the nominal value for the standard pediatric abdominal protocol. Virtual mono-energetic, iodine density, effective atomic number, and electron density results were quantified and compared to their expected values for all acquisition settings and phantom sizes.

Results DECT scans at $100 \mathrm{kVp}$ provided highly accurate spectral results; however, a size dependence was observed for iodine quantification. For the medium phantom configuration ( $15 \mathrm{~cm}$ diameter), measurement errors in iodine density, effective atomic number, and electron density (ED) were below $0.3 \mathrm{mg} / \mathrm{ml}, 0.2$ and $1.8 \% \mathrm{ED}_{\text {water, }}$, respectively. The average accuracy was slightly different from scans at $120 \mathrm{kVp}$; however, not statistically significant for all configurations. Collimation width had no substantial impact. Spectral results were accurate and reliable for radiation exposures down to $0.9 \mathrm{mGy}^{\mathrm{CTDI}} \mathrm{vol}_{\text {ol }}$.

Conclusions Detector-based DECT at $100 \mathrm{kVp}$ can provide on-demand or retrospective spectral information with high accuracy even at extremely low doses, thereby making it an attractive solution for pediatric imaging.
\end{abstract}

Keywords Tomography, X-ray computed · Radiation dose $\cdot$ Phantoms, Imaging

\section{Key points}

- Dual-layer spectral CT at $100 \mathrm{kVp}$ enables high-quality spectral imaging for smaller patients

- Larger collimation width does not affect the accuracy of spectral results

- Accurate and reliable spectral quantification is achievable at radiation doses down to $0.9 \mathrm{mGy}$

\section{Introduction}

Dual-energy CT (DECT) [1-3], the first clinical realization of spectral imaging, is an imaging technology that enables unique quantification of clinically relevant materials based on their elemental composition. By utilizing data from two different $x$-ray spectra, DECT overcomes several limitations of conventional single-energy CT imaging. Dedicated decomposition algorithms that exploit the material-specific energy dependency of $x$-ray attenuation [2, 4-6] provide energy- and material-selective reconstructions. These include virtual mono-energetic images (VMIs) [7], quantitative material density assessment and virtual subtraction of clinical contrast agents, as well as direct information on electron density (ED) and effective atomic number $\left(Z_{\text {eff }}\right)[8]$. 
Multiple solutions have been developed for generating spectral data based on different detection and source technologies [1, 2]. Among the commercially available DECT systems, dual-layer spectral CT has the unique property of creating the spectral separation at the detector level. This design uses a single $x-$ ray source and two attached, but optically separated, scintillation detectors with different sensitivity profiles. While the upper (inner) layer selectively absorbs low-energy $x$-rays and serves as filtration, the bottom (outer) layer is more sensitive to higher energy $x$-rays $[9,10]$.

This detector-based spectral data collection provides multiple advantages. First, both spectral and conventional anatomical images are generated simultaneously for every scan. Hence, one does not need to prospectively select studies for spectral acquisitions and the original imaging workflow can be maintained. Spectral imaging has only been possible at 120 and $140 \mathrm{kVp}$, but the latest generation of detector-based DECT also enables acquisitions at $100 \mathrm{kVp}$. Detector-based spectral separation also provides perfect spatial and temporal alignment of both spectra, enabling efficient projection-space decomposition with an intrinsic reduction of beam-hardening artefacts [7] and better spectral denoising compared to image-space decomposition [9].

While the general clinical benefit of spectral information is well established [11-13], there is less evidence concerning the potential of DECT for pediatric imaging. Current applications of DECT in children include improved automated bone removal for CT angiography, assessment of lung blood volume as a surrogate for perfusion imaging, reduction of metal artifacts originating from highlyattenuating materials such as orthopedic hardware, and diagnosis of abdominal neoplasms [14-16].

One reason for the limited use of DECT in pediatric imaging are the unique requirements of this patient population. First, radiation exposure from medical procedures is a paramount concern for children and must be kept as low as reasonably achievable [17]. Due to their longer lifetime expectancy and increased radiosensitivity of their cells compared to adults, children have a higher cumulative risk of developing radiation-induced cancers. Thus, pediatric CT protocols routinely use lower x-ray beam energies of $100 \mathrm{kVp}$ for abdomen and chest scans to reduce the radiation exposure while maintaining or even improving diagnostic image quality [18]. Second, young children are often unable to comply with instructions and do not remain still during extended scan times, which increases the likelihood for motion artifacts [19]. Using larger collimation widths and fast gantry rotation times, down to 0.27 seconds, allows reduction of the overall acquisition time, potentially avoiding the need for sedation.

The aim of this work is a quantitative investigation of the impact of using $100 \mathrm{kVp}$ tube voltage and $8 \mathrm{~cm}$ beam collimation width on the spectral imaging performance for pediatric imaging using a state-of-theart dual-layer DECT system.

\section{Methods}

\section{Phantom configuration}

In this study, we used a spectral CT phantom (QRM $\mathrm{GmbH}$ ) with custom-made extension rings. The cylindrical $10 \mathrm{~cm}$ diameter phantom (Figure 1) is composed of water-equivalent plastic and contains tissue-equivalent inserts corresponding to liver, adipose, and 100 and $400 \mathrm{mg} / \mathrm{ml}$ hydroxyapatite (HA) materials. For iodine density quantification, four solid iodine inserts with properties equivalent to iodinated contrast media at concentrations of $0.5,2,5$ and $10 \mathrm{mg} / \mathrm{ml}$ were also included. To incorporate the large variability of body size in children and adolescents and assess its impact on spectral quantification, two supplemental extension rings of 15 and $20 \mathrm{~cm}$ outer diameter were 3D-printed from a polylactate acid (PLA) filament. The rings were attached to the original phantom (Figure 1) and the selected diameters $(10,15$ and $20 \mathrm{~cm})$ reflect typical waist circumferences in children aged 2 to 18 years $[20,21]$. 
medRxiv preprint doi: https://doi.org/10.1101/2022.02.27.22271573; this version posted February 28, 2022. The copyright holder for this preprint (which was not certified by peer review) is the author/funder, who has granted medRxiv a license to display the preprint in perpetuity.

All rights reserved. No reuse allowed without permission.

To evaluate the accuracy of the spectral results, the expected (i.e., ground truth) values for all inserts were calculated from the manufacturer material composition specifications. Nominal Hounsfield unit (HU) values for a virtual monoenergetic images (VMI) of energy $E$ are calculated as:

$$
H U(E)=1000 \times\left(\frac{\rho \sum_{i} w_{i} \times \mu_{i}(E)}{\mu_{\text {water }}(E)}-1\right)
$$

where $i$ ranges over the elements composing the material and $w_{i}$ is the normalized mass fraction of element $Z_{i}$ with atomic mass $A_{i}$. $\rho$ represents the physical density and the mass attenuation coefficients ( $\mu_{i}$ and $\mu_{\text {water }}$ for element $i$ and water, respectively) were obtained from the NIST database [22]. The effective atomic number of an insert material is given by $[8,23]$ :

$$
Z_{\mathrm{eff}}=\sqrt[2.94]{\sum_{i} f_{i} Z_{i}^{2.94}}
$$

$f_{i}$ corresponds to the fractional number of electrons of the constituent element $Z_{i}$. The exponent 2.94 is selected based on published literature [8]. Electron density (ED) values provided by the scanner are expressed as percentage relative to water $\left(E D_{w}=3.343 \times 10^{23} \mathrm{~m}^{-3}\right)$ and can be calculated as:

$$
E D=100 \times \frac{N_{A} \rho}{E D_{\mathrm{w}}} \sum_{i} \frac{w_{i}}{A_{i}} Z_{i}
$$

where $N_{A}$ is Avogadro's constant. The nominal values for all insert materials used in this work can be found in Shapira et al. [23].
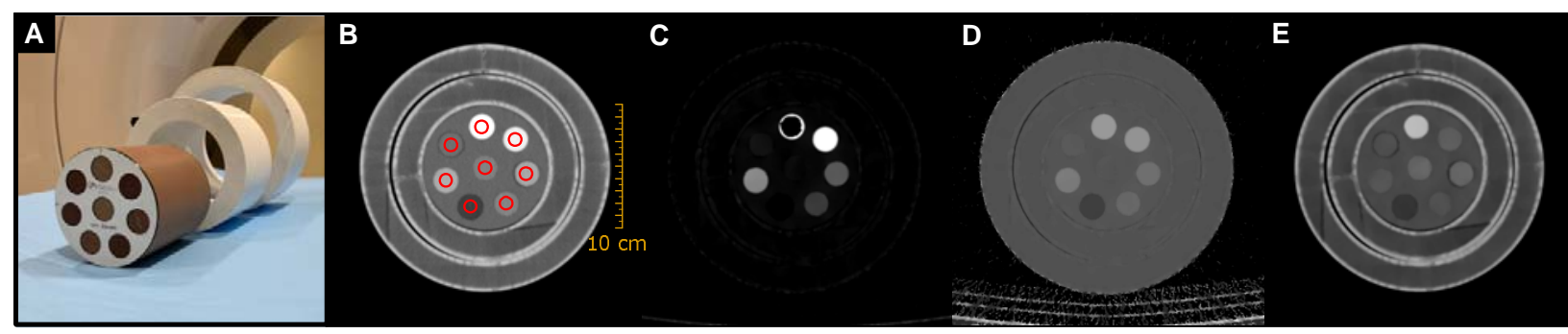

Figure 1 - (A) Spectral CT phantom consisting of a central part with eight inserts and two removable extensions rings. (B) Virtual monoenergetic image at $70 \mathrm{keV}$ (window level/width $=50 / 500 \mathrm{HU}$ ) including regions of interest for image quality evaluations, (C) iodine density map (window level $/$ width $=5 / 10 \mathrm{mg} / \mathrm{ml}$ ), (D) effective atomic number map (window level/width $=10 / 10$ ), and $(E)$ electron density map (window level $/$ width $=110 / 50 \% E D_{\text {water }}$ ) images are shown.

\section{Data acquisition}

All acquisitions were performed on a commercial dual-layer spectral CT scanner (Spectral CT 7500, Philips Healthcare). Scans used the default pediatric $(0-10 \mathrm{~kg})$ abdomen protocol with 0.272 second gantry rotation time and a pitch of 1.234 . Acquisitions were performed at 120 and $100 \mathrm{kVp}$ tube 
voltages as well as 4 and $8 \mathrm{~cm}$ (total) collimation widths. The phantom was positioned in the iso-center and a similar positioning was maintained for all three phantom configurations. For each scenario (i.e., combination of scan parameter and phantom size), images were obtained at four different dose levels $\left(\mathrm{CTDI}_{\text {vol }}\right.$ ) of 9 (default pediatric abdominal protocol), 6, 3 and $0.9 \mathrm{mGy}$. Three repetitions were performed for each scan.

The image series were reconstructed with the hybrid iterative reconstruction method iDose ${ }^{4}$ (spectral denoising level of 2) and a clinical standard kernel for body imaging (B). In total, 48 datasets ( 2 tube voltages $\times 4$ dose levels $\times 2$ collimation widths $\times 3$ repetitions) were collected for each phantom configuration. We did not investigate different spectral denoising levels as they have no substantial impact on the accuracy of spectral results $[8,24]$. The resulting spectral base images contain all relevant information to generate VMls, as well as iodine density, $Z_{\text {eff, }}$ and ED images. A summary of all acquisition and reconstruction parameters is presented in Table 1.

Table 1 - DECT acquisition and reconstruction parameters.

\begin{tabular}{|c|c|}
\hline Tube voltage [kVp] & 120,100 \\
\hline Collimation width [mm] & $40(64 \times 0.625), 80(128 \times 0.625)$ \\
\hline Dose level $\left(\mathrm{CTDI}_{\mathrm{vol}}\right)[\mathrm{mGy}]$ & $9,6,3,0.9$ \\
\hline $\begin{array}{l}\text { Tube current exposure } \\
\text { time product }[\mathrm{mAs}]\end{array}$ & $\begin{array}{l}\text { - } 120 \mathrm{kVp} / 40 \mathrm{~mm}: 109,72,36,11 \\
\text { - } 120 \mathrm{kVp} / 80 \mathrm{~mm}: 116,78,39,11 \\
\text { - } 100 \mathrm{kVp} / 40 \mathrm{~mm}: 175,116,58,17 \\
\end{array}$ \\
\hline Pitch & 1.234 \\
\hline Rotation time [s] & 0.272 \\
\hline Slice thickness [mm] & 3 \\
\hline Field-of-view $\left[\mathrm{mm}^{2}\right]$ & $250 \times 250$ \\
\hline Pixel spacing [mm] & 0.488 \\
\hline
\end{tabular}

\section{Image quality assessment}

Spectral accuracy was measured using circular regions of interest (ROIs) within each cylindrical insert on 26 consecutive axial slices in the center of the phantom. For each configuration, ROls were positioned manually on the high dose $70 \mathrm{keV}$ VMls and covered only half of the actual insert radius to avoid inaccuracies originating from partial volume effects (Figure 1). Subsequently, these ROls were used to extract pixel data from all datasets, i.e., conventional, VMIs, iodine density, $Z_{\text {eff }}$ and ED, and the mean value was obtained for each slice. Statistical analysis was performed using MATLAB R2021b (MathWorks). Differences in spectral quantification accuracy (i.e., mean error) for different collimation widths were compared using Mann-Whitney $U$ tests. The Kruskal-Wallis test was used to compare different dose levels and phantom sizes. In addition, we analyzed changes in spectral quantification variation (i.e., standard deviation of the measurement error) for different dose levels using KruskalWallis tests followed by a post hoc pairwise comparison with Dunn's test (using the Šidák correction). In all cases a $p$-value $\leq 0.05$ was considered as statistically significant.

\section{Results}

We first present each individual spectral result at the pediatric abdominal protocol nominal radiation dose (9 $\left.\mathrm{mGy} \mathrm{CTDI}_{\mathrm{vol}}\right)$. The impact of dose reduction was evaluated separately in the last subsection.

\section{Virtual mono-energetic images}


The accuracy of various VMIs (from $40 \mathrm{keV}$ to $200 \mathrm{keV}$ ) is summarized in Table 2 for scans using the wide $(8 \mathrm{~cm})$ collimation width at 100 and $120 \mathrm{kVp}$. For both tube voltages, VMI HU values tend to increase with increasing phantom size. The average accuracy over all insert materials was 1.3, 3.4, and $5.9 \mathrm{HU}$ for the small, medium, and large phantom configuration at $100 \mathrm{kVp}$. The corresponding results at $120 \mathrm{kVp}$ were 1.2, 2.2, and 4.5 HU. However, differences between both tube voltages were not statistically significant $(p=0.86)$. No difference between 4 and $8 \mathrm{~cm}$ collimation width was observed.

Table 2 - Size dependence of the accuracy of virtual mono-energetic images (VMIs) for the small, medium, and large phantom configurations. The table lists mean error and standard deviation in Hounsfield units (HU). Results are reported for 100 and $120 \mathrm{kVp}$ scans at a collimation width of $8 \mathrm{~cm}$. Negative and positive errors indicate an under- and overestimation of the nominal value, respectively.

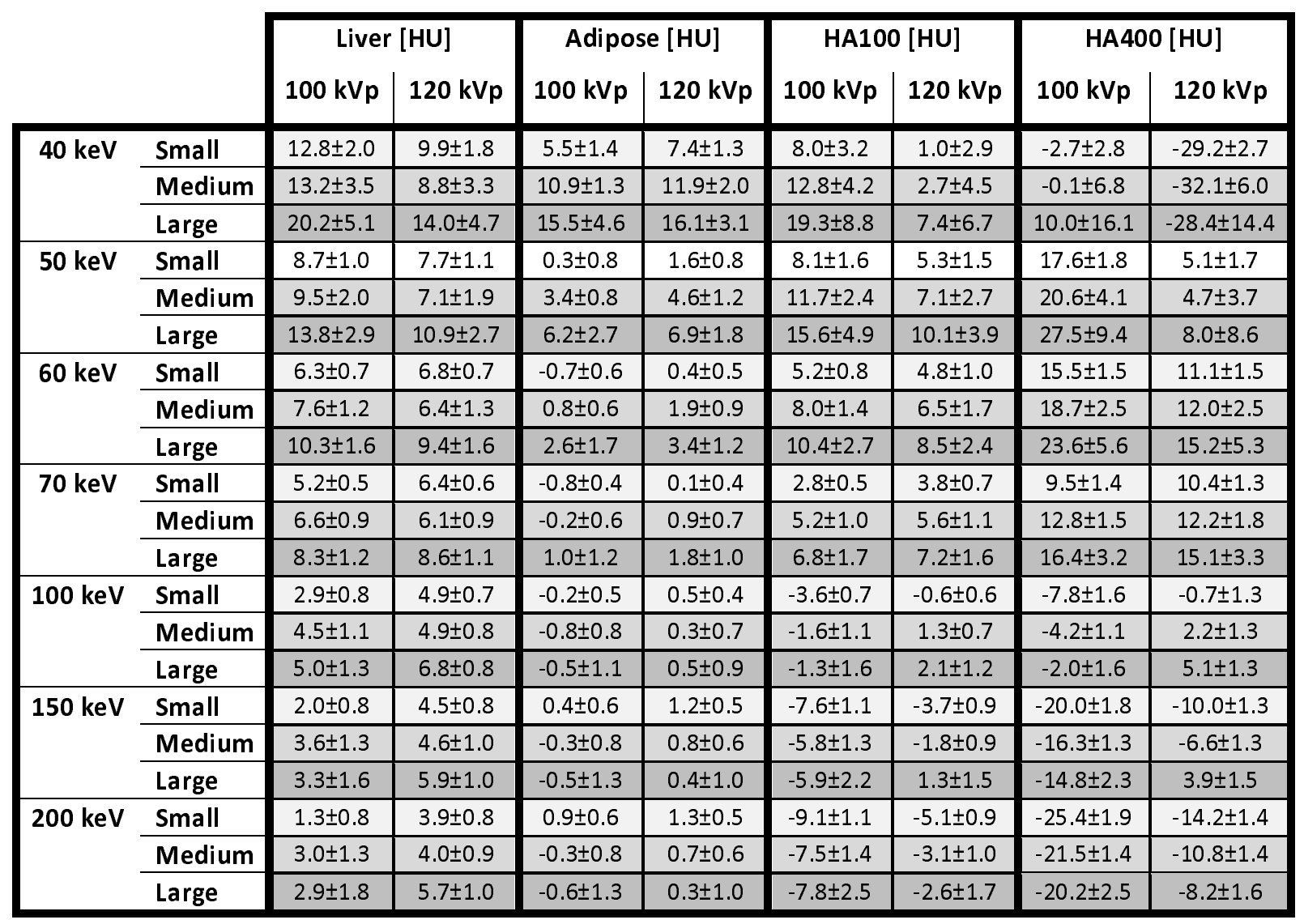

A VMI of $62 \mathrm{keV}$ was selected for further comparison, as it most closely matched the HU values obtained from the conventional $100 \mathrm{kVp}$ data for all phantom sizes. Figure 2 shows the absolute HU difference from the average value over the three phantom sizes for conventional and $62 \mathrm{keV} \mathrm{VMI}$ data. For the medium phantom size $(15 \mathrm{~cm})$, both conventional and VMI provide a comparably low deviation of less than $3 \mathrm{HU}$. The VMI accuracy for the small and large phantom configuration is only slightly degraded. In contrast, conventional images demonstrate a substantially larger size-dependence with CT number variations of up to $28 \mathrm{HU}$. 
medRxiv preprint doi: https://doi.org/10.1101/2022.02.27.22271573; this version posted February 28, 2022. The copyright holder for this preprint (which was not certified by peer review) is the author/funder, who has granted medRxiv a license to display the preprint in perpetuity.

All rights reserved. No reuse allowed without permission.

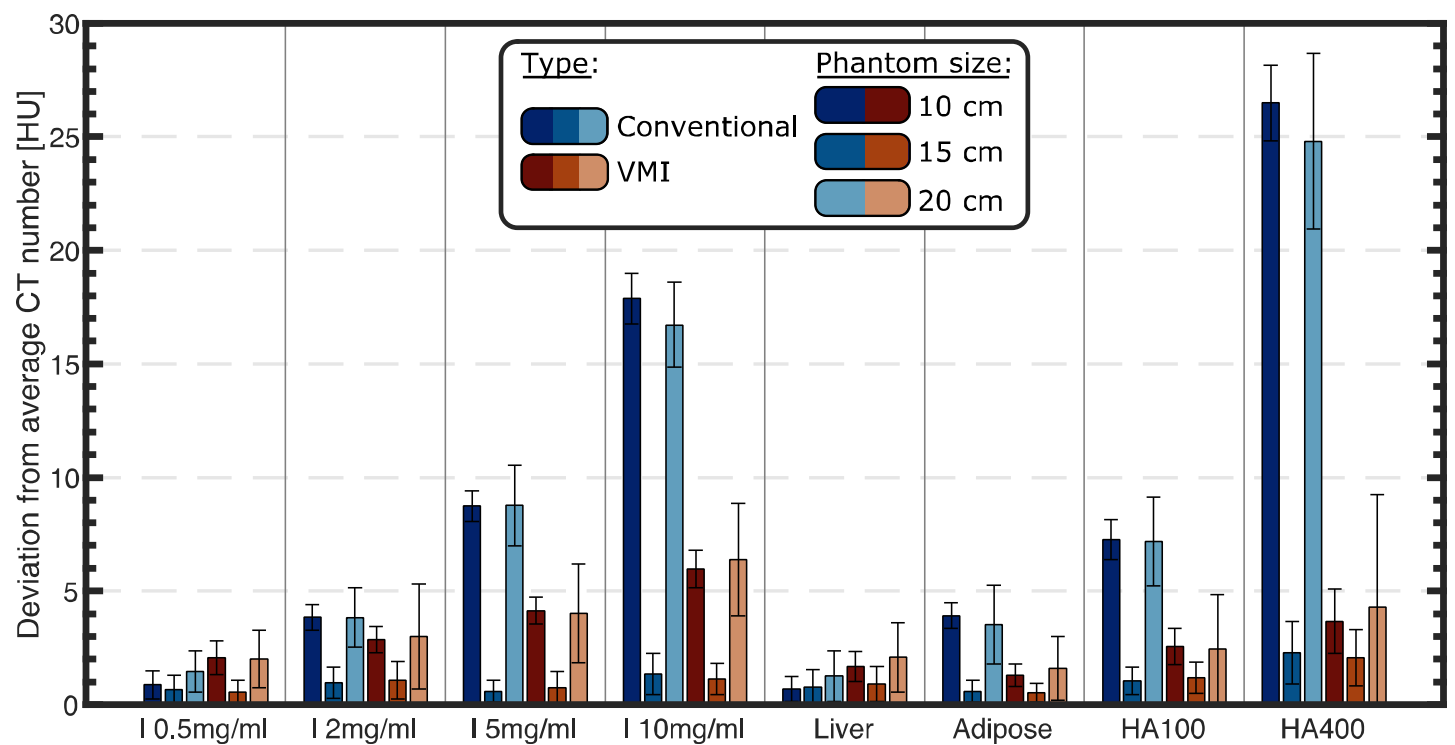

Figure 2 - Bar plot of the (absolute) HU deviation from the size-averaged value for the conventional $100 \mathrm{kVp}$ (blue) and the corresponding $62 \mathrm{keV}$ virtual mono-energetic images (VMls; red) data. The bars and whiskers represent the mean error and standard deviation, respectively. Different phantom sizes are displayed in different shades.

\section{2. lodine density}

The error in quantified iodine density is shown in Figure 3 for inserts of increasing iodine concentration. A significant size dependence was observed for both tube voltages. Larger variations occurred for higher iodine concentrations and for smaller phantom geometries. $100 \mathrm{kVp}$ yields slightly higher absolute iodine density errors of $0.17,0.07$, and $0.04 \mathrm{mg} / \mathrm{ml}$ compared to $120 \mathrm{kVp}$ for the small, medium, and large phantom sizes, respectively. However, those differences were not significant for any configuration. Moreover, no significant difference in measurement error was found when comparing 4 and $8 \mathrm{~cm}$ collimation widths.

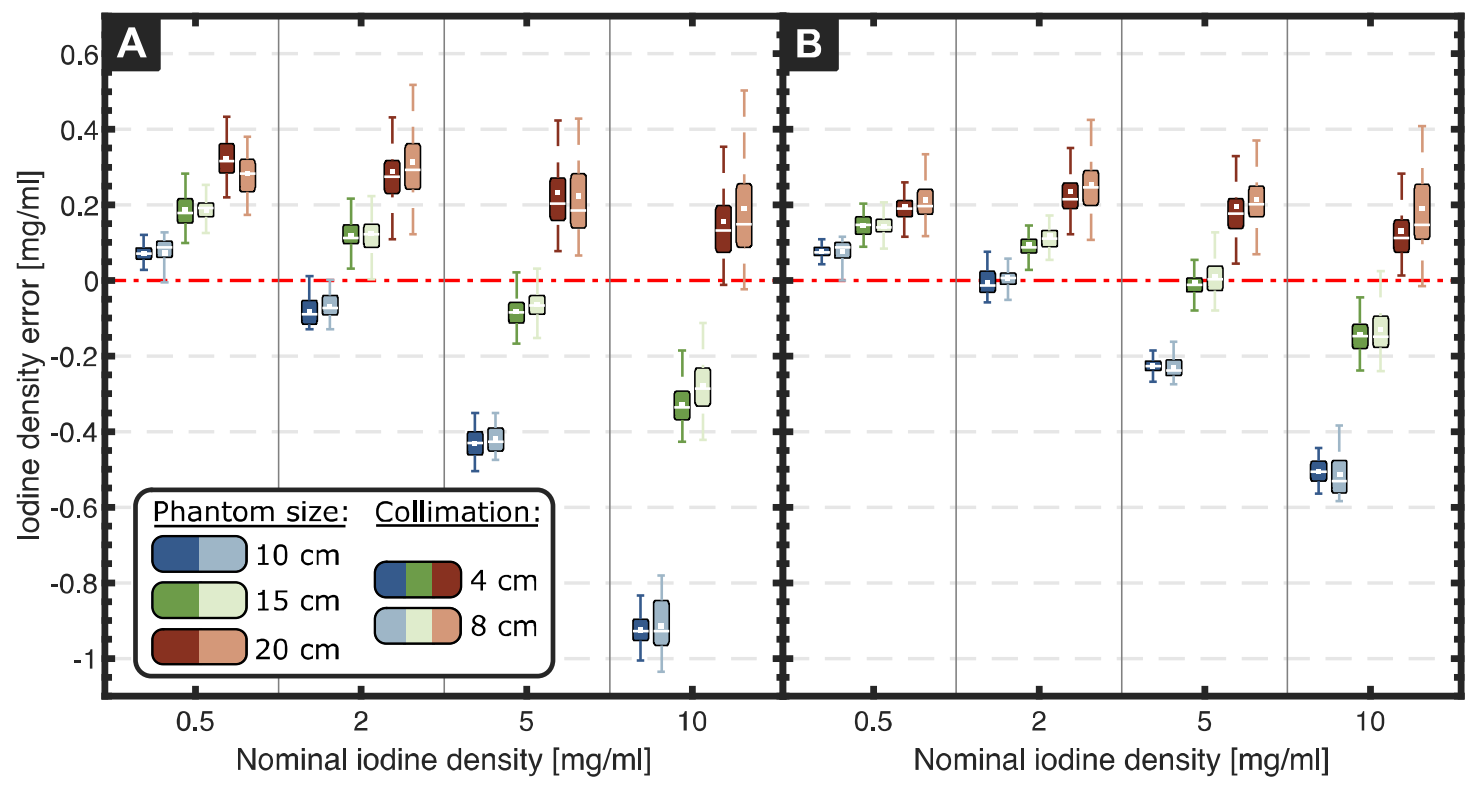

Figure 3 - lodine density errors for scans acquired at (A) $100 \mathrm{kVp}$ and (B) $120 \mathrm{kVp}$. Mean and median of the distribution are represented by the central white line and marker, respectively, the box corresponds to the first 
and third quartile, and each whisker indicates 1.5 times the interquartile range. Different phantom sizes and collimation widths are displayed in different colors and shades, respectively.

\section{Effective atomic number}

Figure 4 shows the $Z_{\text {eff }}$ errors obtained for the various acquisition and phantom configurations. Overall, the size-dependence is much smaller than for iodine density (Figure 3), except for adipose tissue. Both tube voltages showed no significant differences when comparing the measurement error among the three phantom sizes ( $100 \mathrm{kVp}: p=0.47,120 \mathrm{kVp}: p=0.72$ ). The mean accuracy was 0.23 and 0.16 for $100 \mathrm{kVp}$ and $120 \mathrm{kVp}$ scans, respectively. No significant difference between both collimation widths was observed.

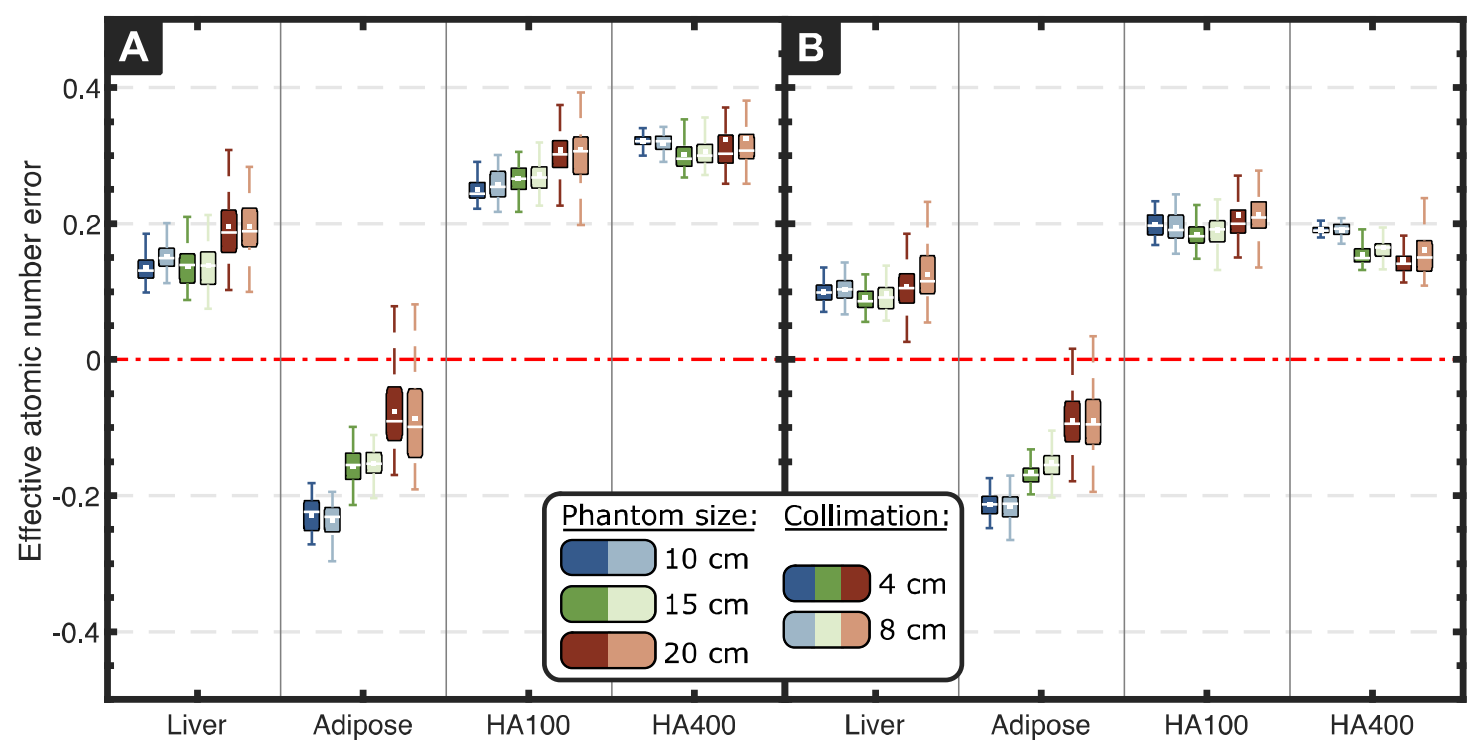

Figure 4 - Effective atomic number $\left(Z_{\text {eff }}\right)$ errors for scans acquired at ( $\left.A\right) 100 \mathrm{kVp}$ and (B) $120 \mathrm{kVp}$. Mean and median of the distribution are represented by the central white line and marker, respectively, the box corresponds to the first and third quartile, and each whisker indicates 1.5 times the interquartile range. Different phantom sizes and collimation widths are displayed in different colors and shades, respectively.

\section{Electron density}

The electron density quantification is displayed in Figure 5. All mean errors were within the uncertainty of the nominal electron density values of $2 \% \mathrm{ED}_{\text {water }}$ except $\mathrm{HA} 400$ for the smallest phantom size at 100 $\mathrm{kVp}$ (2.1\%ED water mean error). As for $Z_{\mathrm{eff}}$, there was no influence of phantom size on measurement error (100 kVp: $p=0.91,120 \mathrm{kVp}: p=0.64$ ). The mean electron density error was 0.72 and $0.34 \% \mathrm{ED}_{\text {water }}$ for $100 \mathrm{kVp}$ and $120 \mathrm{kVp}$, with the largest differences occurring for HA400. 


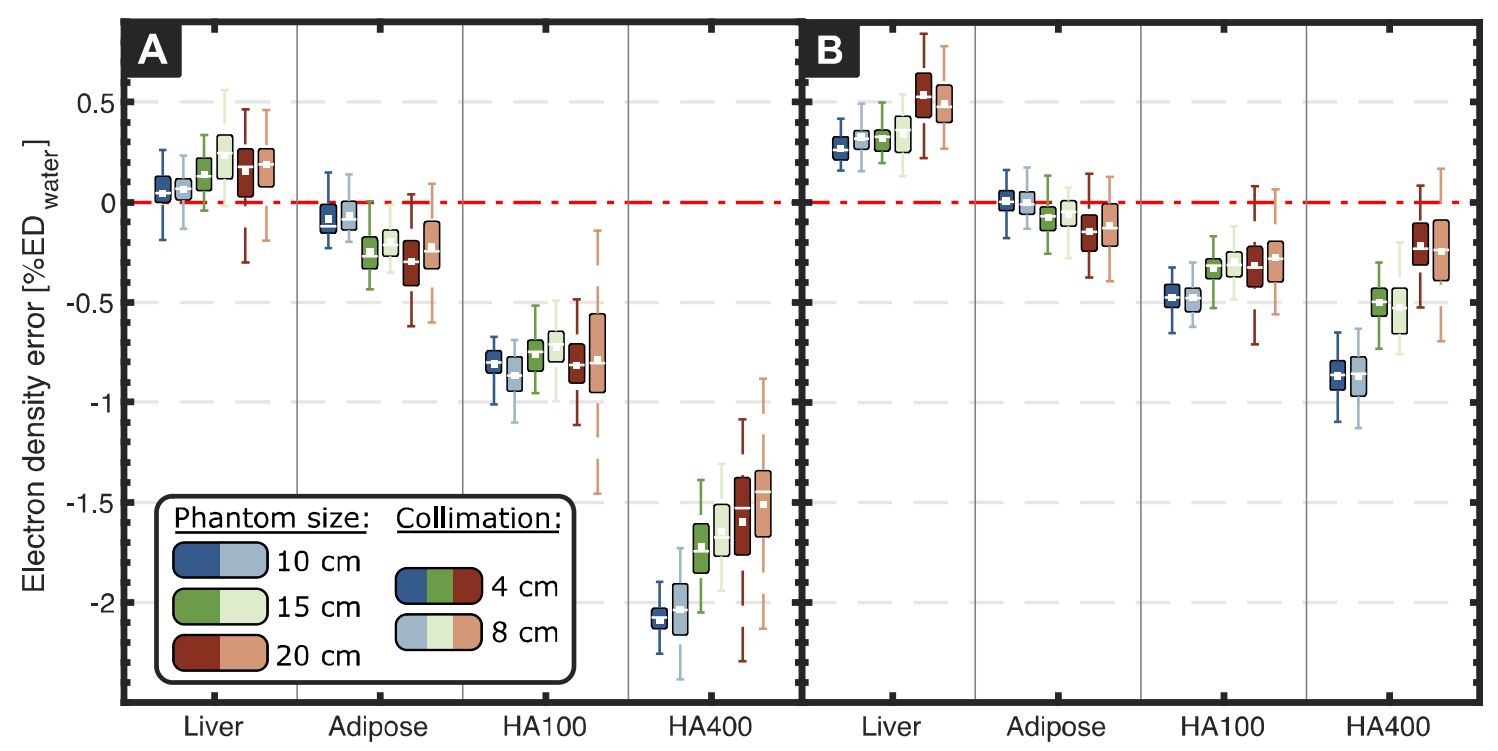

Figure 5 - Electron density (ED) errors for scans acquired at (A) $100 \mathrm{kVp}$ and (B) $120 \mathrm{kVp}$. The uncertainty of the nominal electron density values is $2 \% \mathrm{ED}_{\text {water }}$. Mean and median of the distribution are represented by the central white line and marker, respectively, the box corresponds to the first and third quartile, and each whisker indicates 1.5 times the interquartile range. Different phantom sizes and collimation widths are displayed in different colors and shades, respectively. No influence of the collimation on the electron density quantification was observed.

\section{Radiation dose dependency}

No significant differences in spectral accuracy (mean measurement errors) were found for both tube potentials and all phantom sizes when comparing results obtained at $0.9,3,6$ and 9 mGy CTDI vol $_{\text {. Figure }}$ 6 shows the standard deviation of the spectral results obtained from all relevant insert materials. As expected, higher values are observed for larger phantom configurations and with lower imaging doses. However, the results obtained at $0.9 \mathrm{mGy}$ were significantly different only for the $20 \mathrm{~cm}$ phantom. The corresponding standard deviations for $120 \mathrm{kVp}$ scans were lower by $0.02 \mathrm{mg} / \mathrm{ml}, 0.01$ and $0.05 \% \mathrm{ED}_{\text {water, }}$, for iodine density, effective atomic number, and electron density, respectively. Significant differences between 4 (not shown) and $8 \mathrm{~cm}$ collimation widths were only observed for iodine density (100 kVp and $120 \mathrm{kVp})$ and electron density (120 kVp), and only with the smallest phantom configuration.
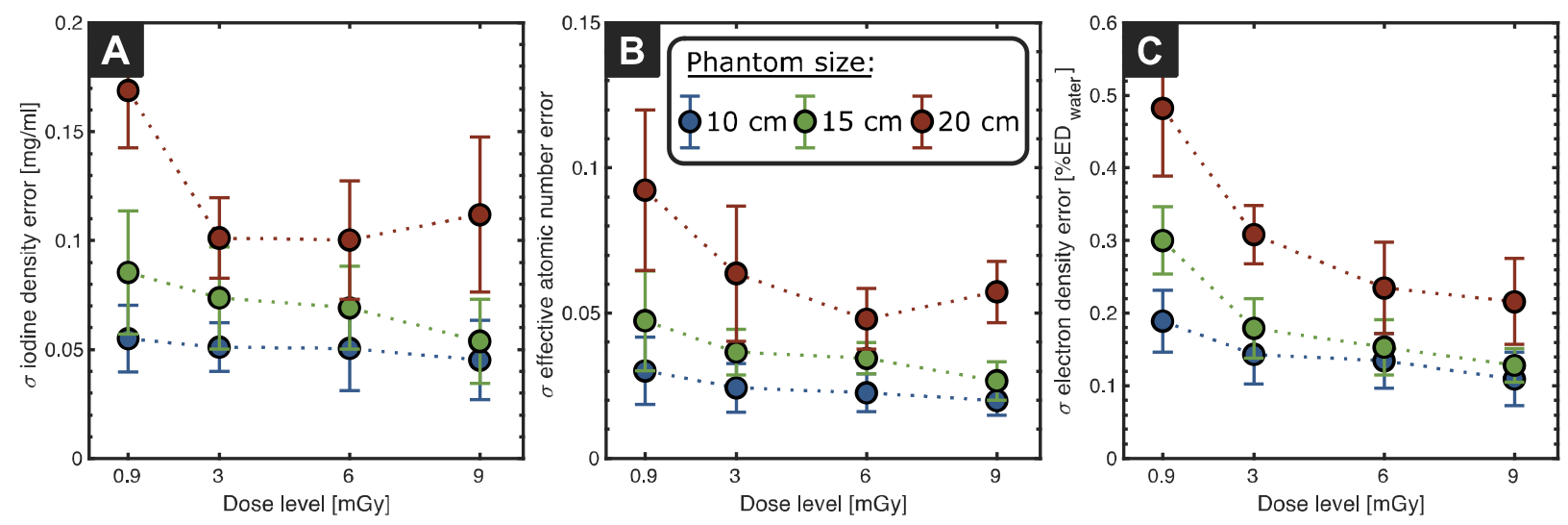

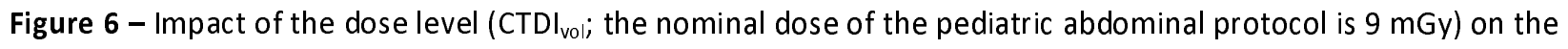
standard deviation of spectral results: (A) iodine density, (B) effective atomic number, and (C) electron density obtained for scans at $8 \mathrm{~cm}$ collimation width. 
medRxiv preprint doi: https://doi.org/10.1101/2022.02.27.22271573; this version posted February 28, 2022. The copyright holder for this preprint (which was not certified by peer review) is the author/funder, who has granted medRxiv a license to display the preprint in perpetuity.

All rights reserved. No reuse allowed without permission.

\section{Discussion}

While spectral imaging is becoming more widely utilized in clinical imaging, few studies have been published on DECT in the pediatric population and more experience is required for its adoption into clinical practice [14]. Precise and reliable spectral quantification is a key requirement of DECT. However, for pediatric imaging additional factors need to be considered including dose and scan time. While larger collimation width and lower tube voltage could be beneficial, they might compromise spectral quantification. We present the first investigation of quantitative spectral imaging performance at 100 $\mathrm{kVp}$ for a dedicated pediatric-sized phantom using the latest generation detector-based DECT.

$62 \mathrm{keV}$ VMls demonstrated very similar attenuation values (within few HUs) compared to conventional $100 \mathrm{kVp}$ images across all insert materials for the medium phantom size (Figure 2). Moreover, VMls substantially increased the stability of $\mathrm{HU}$ values for varying patient geometries, which can be attributed to accurate correction of beam hardening effects $[23,25]$. Hence, VMls can enable consistent attenuation quantification for pediatric patients despite the variability of body sizes occurring during childhood. This can improve the characterization and increase conspicuity of abdominal pathologies [16].

lodine quantification was affected by the phantom size (Figure 3). Similar findings have been reported for previous generations of dual layer [23, 26] and dual source DECT [27]. Overall, measured iodine densities systematically increased with phantom size and changes were larger for higher iodine concentrations. However, the error was always within $1 \mathrm{mg} / \mathrm{ml}$. Hence, radiologists need to consider patient size when interpreting images based on quantitative iodine density information. Future work should aim to improve the stability of material decomposition algorithms or develop adequate correction methods.

Effective atomic number and electron density results showed excellent accuracy (Figures 4 and 5) and were less sensitive to phantom size. This can be utilized to improve the relative stopping power accuracy in proton therapy treatment planning [28], which faces inherent limitations when relying on conventional CT images. Proton therapy allows sparing of normal tissues and reduces the integral dose, thereby making it very appealing for radiotherapy treatment in children [29].

Scans acquired at $120 \mathrm{kVp}$ provided slightly better accuracy than $100 \mathrm{kVp}$, which can be attributed to the reduced spectral separation at lower tube potentials, as has been reported by others for $120 / 140$ $k V p[30,31]$. However, it should be noted that results depend on phantom size and attenuation [26]. The collimation width did not significantly affect spectral results (Figures 3-5). A larger collimation not only improves dose utilization but also reduces scan time, both crucial aspects for pediatric patients. It should also be noted that scans in this study were acquired with a very fast rotation time of $0.272 \mathrm{~s}$.

Compared with its predecessor (IQon Spectral CT, Philips Healthcare), the overall spectral accuracy was improved [23]. For a comparable setting (medium phantom size, $9 \mathrm{mGy}$ radiation dose, and $4 \mathrm{~cm}$ collimation width) the absolute error in VMI CT number, effective atomic number, and electron density are reduced by $8.69 \mathrm{HU}, 0.03$ and $0.45 \% \mathrm{ED}_{\text {water, }}$ respectively, and the iodine density accuracy is comparable. The quantitative improvements between the two systems can be attributed to two technological advancements. First, the newer CT 7500 is equipped with a next-generation dual-layer $x-$ ray detection system that is based on a higher-efficiency detector array coupled with a two-dimensional anti-scatter grid. In contrast, the previous generation detector array was based on a stick design that readout the scintillator from the side and necessitated a one-dimensional anti-scatter grid [10]. A second reason for the improvements in spectral quantification is an updated physics model of the detector system that is utilized in the 2-base material decomposition process. These improvements include more accurate detector design inputs and the inclusion of second-order physics effects, i.e., light-matter interactions, that occur within the detector system.

To the best of our knowledge there are no dedicated studies comparing the imaging performance of different DECT implementations for pediatric imaging. While many have compared the imaging 
performance of different DECT technologies in larger phantoms [32, 33], results might differ for pediatric-sized phantoms. Moreover, the recent clinical implementation of the first commercial photocounting CT [34-36] may open new possibilities for spectral imaging that can benefit pediatric patients; however, future studies are needed to investigate and compare the pediatric imaging performance.

The findings of our work evidence the high image quality achievable using standard pediatric abdominal protocol doses. The use of lower tube voltage and dedicated CT protocols are critical to avoid high effective doses due to the smaller transverse body diameter in children, which highlights the need for a careful trade-off between dose and image quality [37]. In fact, it has been demonstrated that DECT scans of the head and abdomen in children can be dose-neutral compared to single energy CT [38, 39]. We found that spectral quantification was robust with further dose reductions down to $10 \%$ of the protocol's nominal value (Figure 6). Variations were slightly higher for larger phantom sizes due to their increased attenuation, which is in line with previous publications [23, 31].

A limitation of our study is the use of a geometric phantom. This enables consistent evaluation of spectral results with known ground truth but does not include anatomical features or organs. Therefore, future investigations should be extended to more realistic scenarios such as pediatric anthropomorphic or 3D printed [40] phantoms (ideally tailored to DECT applications). We also only employed a single scan protocol. However, results are not expected to vary much with scan parameters due to the simultaneous acquisition of both spectra [23].

Spectral imaging enables characterization of materials based on their attenuation at different energies and has resulted in various novel applications of $C T$ imaging within the last two decades. Our initial phantom evaluation has shown that detector-based DECT imaging at $100 \mathrm{kVp}$ maintains a high quality of spectral accuracy. Even though DECT offers several clear advantages, further clinical studies are needed for the broad integration of spectral information into pediatric imaging due to its unique requirements.

\section{Acknowledgment}

The authors acknowledge support through the National Institutes of Health (R01EB030494). In addition, the authors would like to thank Michael Geagan for his help with 3D-printing the extension rings and Safaa Abdallah for her help with acquiring the data.

\section{Conflict of interest}

Harold I. Litt and Peter B. Noël received a hardware grant from Philips Healthcare. Peter B. Noël receives research grant funding from Philips Healthcare. Dr. Halliburton is an employee of Philips Healthcare. The other authors have no relevant conflicts of interest to disclose.

\section{References}

1. Johnson TRC (2012) Dual-energy CT: general principles. AJR Am J Roentgenol 199:3-8. https://doi.org/10.2214/ajr.12.9116

2. McCollough CH, Leng S, Yu L, Fletcher JG (2015) Dual-and Multi-Energy CT. RSNA Radiol 276:637653

3. McCollough CH, Boedeker K, Cody D, et al (2020) Principles and applications of multienergy CT: Report of AAPM Task Group 291. Med Phys 47:e881-e912. https://doi.org/10.1002/mp.14157

4. Alvarez RE, Macovski A (1976) Energy-selective reconstructions in \{X-ray\} computerised tomography. Phys Med Biol 21:733-744

5. Johnson TRC, Krauß B, Sedlmair M, et al (2007) Material differentiation by dual energy CT: Initial experience. Eur Radiol 17:1510-1517. https://doi.org/10.1007/s00330-006-0517-6

6. Maaß C, Baer M, Kachelrieß M (2009) Image-based dual energy CT using optimized precorrection functions: A practical new approach of material decomposition in image domain. Med Phys 36:3818-3829. https://doi.org/10.1118/1.3157235 
medRxiv preprint doi: https://doi.org/10.1101/2022.02.27.22271573; this version posted February 28, 2022. The copyright holder for this preprint (which was not certified by peer review) is the author/funder, who has granted medRxiv a license to display the preprint in perpetuity.

All rights reserved. No reuse allowed without permission.

7. Yu L, Leng S, McCollough CH (2012) Dual-energy CT-based monochromatic imaging. AJR Am J Roentgenol 199:9-15. https://doi.org/10.2214/ajr.12.9121

8. Hua CH, Shapira N, Merchant TE, et al (2018) Accuracy of electron density, effective atomic number, and iodine concentration determination with a dual-layer dual-energy computed tomography system. Med Phys 45:2486-2497. https://doi.org/10.1002/mp.12903

9. Hokamp NG, Maintz D, Shapira N, et al (2020) Technical background of a novel detector-based approach to dual-energy computed tomography. Diagnostic Interv Radiol 26:68-71. https://doi.org/10.5152/dir.2019.19136

10. Shefer E, Altman A, Behling R, et al (2013) State of the Art of CT Detectors and Sources: A Literature Review. Curr Radiol Rep 1:76-91. https://doi.org/10.1007/s40134-012-0006-4

11. Patino M, Prochowski A, Agrawal MD, et al (2016) Material separation using dual-energy CT: Current and emerging applications. Radiographics 36:1087-1105.

https://doi.org/10.1148/rg.2016150220

12. Albrecht MH, Vogl TJ, Martin SS, et al (2019) Review of clinical applications for virtual monoenergetic dual-energy CT. Radiology 293:260-271.

https://doi.org/10.1148/radiol.2019182297

13. Silva AC, Morse BG, Hara AK, et al (2011) Dual-energy (spectral) CT: Applications in abdominal imaging. Radiographics 31:1031-1046. https://doi.org/10.1148/rg.314105159

14. Siegel MJ, Ramirez-Giraldo JC (2019) Dual-energy CT in children: Imaging algorithms and clinical applications. Radiology 291:286-297. https://doi.org/10.1148/radiol.2019182289

15. Rapp JB, Biko DM, Barrera CA, et al (2020) Current and Future Applications of Thoracic DualEnergy CT in Children: Pearls and Pitfalls of Technique and Interpretation. Semin Ultrasound, CT MRI 41:433-441. https://doi.org/10.1053/j.sult.2020.05.008

16. Kamps SE, Otjen JP, Stanescu AL, et al (2020) Dual-Energy CT of Pediatric Abdominal Oncology Imaging: Private Tour of New Applications of CT Technology. 967-975

17. Voss SD, Reaman GH, Kaste SC, Slovis TL (2009) The ALARA concept in pediatric oncology. Pediatr Radiol 39:1142-1146. https://doi.org/10.1007/s00247-009-1404-5

18. Nievelstein RAJ, Van Dam IM, Van Der Molen AJ (2010) Multidetector CT in children: Current concepts and dose reduction strategies. Pediatr Radiol 40:1324-1344.

https://doi.org/10.1007/s00247-010-1714-7

19. Bastos M d'Almeida, Lee EY, Strauss KJ, et al (2009) Motion Artifact on High-Resolution CT Images of Pediatric Patients: Comparison of Volumetric and Axial CT Methods. Am J Roentgenol 193:1414-1418. https://doi.org/10.2214/AJR.09.2843

20. Fernández JR, Bohan Brown M, López-Alarcón M, et al (2017) Changes in pediatric waist circumference percentiles despite reported pediatric weight stabilization in the United States.

Pediatr Obes 12:347-355. https://doi.org/10.1111/ijpo.12150

21. Fernández JR, Redden DT, Pietrobelli A, Allison DB (2004) Waist circumference percentiles in nationally representative samples of African-American, European-American, and MexicanAmerican children and adolescents. J Pediatr 145:439-444.

https://doi.org/10.1016/j.jpeds.2004.06.044

22. Berger MJ, Hubbell JH, Seltzer SM, et al (1998) XCOM: photon cross sections database. NIST Stand Ref Database

23. Shapira N, Mei K, Noël PB (2021) Spectral CT quantification stability and accuracy for pediatric patients: A phantom study. J Appl Clin Med Phys 22:16-26. https://doi.org/10.1002/acm2.13161

24. Lu X, Lu Z, Yin J, et al (2019) Effects of radiation dose levels and spectral iterative reconstruction levels on the accuracy of iodine quantification and virtual monochromatic CT numbers in duallayer spectral detector CT: An iodine phantom study. Quant Imaging Med Surg 9:188-200. https://doi.org/10.21037/qims.2018.11.12 
medRxiv preprint doi: https://doi.org/10.1101/2022.02.27.22271573; this version posted February 28, 2022. The copyright holder for this preprint (which was not certified by peer review) is the author/funder, who has granted medRxiv a license to display the preprint in perpetuity.

All rights reserved. No reuse allowed without permission.

25. D'Angelo T, Cicero G, Mazziotti S, et al (2019) Dual energy computed tomography virtual monoenergetic imaging: technique and clinical applications. Br J Radiol 20180546. https://doi.org/10.1259/bjr.20180546

26. Van Hedent S, Tatsuoka C, Carr S, et al (2020) Impact of Patient Size and Radiation Dose on Accuracy and Precision of lodine Quantification and Virtual Noncontrast Values in Dual-layer Detector CT-A Phantom Study. Acad Radiol 27:409-420.

https://doi.org/10.1016/j.acra.2019.02.013

27. Jiang X, Yang X, Hintenlang DE, White RD (2021) Effects of Patient Size and Radiation Dose on lodine Quantification in Dual-Source Dual-Energy CT. Acad Radiol 28:96-105.

https://doi.org/10.1016/j.acra.2019.12.027

28. Bär E, Collins-Fekete C, Rompokos V, et al (2021) Assessment of the impact of CT calibration procedures for proton therapy planning on pediatric treatments. Med Phys 48:5202-5218. https://doi.org/10.1002/mp.15062

29. Leroy R, Benahmed N, Hulstaert F, et al (2016) Proton Therapy in Children: A Systematic Review of Clinical Effectiveness in 15 Pediatric Cancers. Int J Radiat Oncol 95:267-278.

https://doi.org/10.1016/j.ijrobp.2015.10.025

30. Pelgrim GJ, van Hamersvelt RW, Willemink MJ, et al (2017) Accuracy of iodine quantification using dual energy CT in latest generation dual source and dual layer CT. Eur Radiol 27:3904-3912. https://doi.org/10.1007/s00330-017-4752-9

31. Sauter AP, Kopp FK, Münzel D, et al (2018) Accuracy of iodine quantification in dual-layer spectral $\mathrm{CT}$ : Influence of iterative reconstruction, patient habitus and tube parameters. Eur J Radiol 102:83-88. https://doi.org/10.1016/j.ejrad.2018.03.009

32. Jacobsen MC, Schellingerhout D, Wood CA, et al (2018) Intermanufacturer Comparison of DualEnergy CT lodine Quantification and Monochromatic Attenuation: A Phantom Study. Radiology 287:224-234. https://doi.org/10.1148/radiol.2017170896

33. Sellerer T, Noël PB, Patino M, et al (2018) Dual-energy CT: a phantom comparison of different platforms for abdominal imaging. Eur Radiol 28:2745-2755. https://doi.org/10.1007/s00330-0175238-5

34. Liu LP, Shapira N, Sahbaee P, et al (2021) First-generation clinical dual-source photon-counting CT: ultra-low dose quantitative spectral imaging. medRxiv 2021.11.17.21266432. https://doi.org/10.1101/2021.11.17.21266432

35. van der Werf NR, van Gent M, Booij R, et al (2021) Dose Reduction in Coronary Artery Calcium Scoring Using Mono-Energetic Images from Reduced Tube Voltage Dual-Source Photon-Counting CT Data: A Dynamic Phantom Study. Diagnostics 11:2192.

https://doi.org/10.3390/diagnostics11122192

36. Rajendran K, Petersilka M, Henning A, et al (2021) First Clinical Photon-counting Detector CT System: Technical Evaluation. Radiology. https://doi.org/10.1148/radiol.212579

37. Goodman TR, Mustafa A, Rowe E (2019) Pediatric CT radiation exposure: where we were, and where we are now. Pediatr Radiol 49:469-478. https://doi.org/10.1007/s00247-018-4281-y

38. Zhu X, McCullough WP, Mecca P, et al (2016) Dual-energy compared to single-energy CT in pediatric imaging: a phantom study for DECT clinical guidance. Pediatr Radiol 46:1671-1679. https://doi.org/10.1007/s00247-016-3668-x

39. Siegel MJ, Curtis WA, Ramirez-Giraldo JC (2016) Effects of Dual-Energy Technique on Radiation Exposure and Image Quality in Pediatric Body CT. Am J Roentgenol 207:826-835. https://doi.org/10.2214/AJR.15.15994

40. Mei K, Geagan M, Roshkovan L, et al (2021) Three-dimensional printing of patient-specific lung phantoms for $\mathrm{CT}$ imaging: Emulating lung tissue with accurate attenuation profiles and textures. Med Phys. https://doi.org/10.1002/mp.15407 
medRxiv preprint doi: https://doi.org/10.1101/2022.02.27.22271573; this version posted February 28, 2022. The copyright holder for this preprint (which was not certified by peer review) is the author/funder, who has granted medRxiv a license to display the preprint in perpetuity.

All rights reserved. No reuse allowed without permission. 